\title{
Higher order mode damping in a five-cell superconducting rf cavity with a photonic band gap coupler cell
}

\author{
Sergey A. Arsenyev* and Richard J. Temkin \\ Massachusetts Institute of Technology (MIT), 77 Massachusetts Avenue, Cambridge, \\ Massachusetts 02139, USA \\ Dmitry Yu. Shchegolkov and Evgenya I. Simakov \\ Los Alamos National Laboratory, P.O. Box 1663, Los Alamos, New Mexico 87545, USA \\ Chase H. Boulware, Terry L. Grimm, and Adam R. Rogacki \\ Niowave, Inc., 1012 North Walnut Street, Lansing, Michigan 48906, USA \\ (Received 17 March 2016; published 1 August 2016)
}

\begin{abstract}
We present a study of higher order mode (HOM) damping in the first multicell superconducting radiofrequency (SRF) cavity with a photonic band gap (PBG) coupler cell. Achieving higher average beam currents is particularly desirable for future light sources and particle colliders based on SRF energyrecovery linacs (ERLs). Beam current in ERLs is limited by the beam breakup instability, caused by parasitic HOMs interacting with the beam in accelerating cavities. A PBG cell incorporated in an accelerating cavity can reduce the negative effect of HOMs by providing a frequency selective damping mechanism, thus allowing significantly higher beam currents. The five-cell cavity with a PBG cell was designed and optimized for HOM damping. Monopole and dipole HOMs were simulated. The SRF cavity was fabricated and tuned. External quality factors for some HOMs were measured in a cold test. The measurements agreed well with the simulations.
\end{abstract}

DOI: 10.1103/PhysRevAccelBeams.19.081301

\section{INTRODUCTION}

Particle colliders and light sources are among the most interesting applications for future accelerator science and technology. Colliders help to push the limits of our understanding of fundamental particle physics, while light sources are used to study condensed matter physics, material science, biology, and medicine. In the past decade, there has been significant interest in energy-recovery-linac (ERL) driver accelerators for the light sources [1-4] and electron-proton colliders [5].

The motivation for using ERLs is their energy efficiency and capability to provide the beam with emittance much smaller than can be attained in a storage-ring-based light source [6]. Energy efficiency is especially important for the projects described in Refs. [2-5] that require electron beams with energies of multiple $\mathrm{GeV}$ and high continuous wave $(\mathrm{CW})$ beam currents on the order of hundreds of milliamperes. Indeed, this means that driver linacs would have to produce a beam power as high as $1 \mathrm{GW}$, which is feasible only if the energy used to accelerate the beam is somehow recovered [6]. ERLs achieve that by decelerating

\footnotetext{
*sergejs@alum.mit.edu

Published by the American Physical Society under the terms of the Creative Commons Attribution 3.0 License. Further distribution of this work must maintain attribution to the author $(s)$ and the published article's title, journal citation, and DOI.
}

electron beams after they have been used for x-ray production or in collisions with another beam, recapturing a big fraction of the energy spent on acceleration and feeding it back to the subsequent beam bunches.

For the projects described in Refs. [2-5], high beam currents of hundreds of milliamperes are needed to increase the brilliance in the light sources or luminosity in the colliders. Even higher currents, up to the ampere level, are desirable for the future projects, as described in Refs. [7,8]. While currents up to the ampere level are possible in ERLs with relatively low electron energies of multiple $\mathrm{MeV}$ [9-11], raising the beam current for a multi-GeV machine is a great challenge. This is due to the fact that multi-GeV ERLs have to be much longer, which makes the beam more vulnerable to instabilities and thus limits the achievable current. The main limitation comes from the beam breakup (BBU) instability, which comes as a result of the interaction of the beam with parasitic electromagnetic modes in accelerating structures. This effect is caused primarily by the modes of dipole or quadrupole nature with frequencies above the accelerating rf [hence, they are called higher order modes (HOMs)].

Consideration of the BBU instability becomes especially important given the fact that many ERLs [1-5] rely on superconducting radio-frequency (SRF) accelerating cavities that are the natural choice for accelerators operating in a $\mathrm{CW}$ mode [12]. Once HOMs are excited in superconducting cavities, they can oscillate for a long time 
with very high $Q$ factors due to extremely low losses in the walls of the cavities.

For ERLs with one recirculating turn, an estimate for the BBU threshold current $I_{\text {th }}$ was derived for the $n$th HOM in Ref. [13]:

$$
I_{\text {th }}=-\frac{2 c^{2}}{e\left(\frac{R}{Q}\right)_{n} Q_{n} \omega_{n}} \frac{1}{T_{12}^{*} \sin \left(\omega_{n} t_{r}\right)},
$$

where the matrix $T$ describes how the beam's transverse momentum translates into the transverse displacement after one turn:

$$
T_{12}^{*}=T_{12} \cos ^{2} \theta_{n}+\left(T_{14}+T_{32}\right) \sin \theta_{n} \cos \theta_{n}+T_{34} \sin ^{2} \theta_{n} .
$$

In Eqs. (1) and (2), $c$ is the speed of light, $e$ is the elementary charge, $(R / Q)_{n}$ is the shunt impedance of the $n$th HOM (in ohms), $Q_{n}$ is its loaded quality factor, $\omega_{n}=2 \pi f_{n}$ is its angular frequency, $\theta_{n}$ is the polarization angle from the $x$ direction, and $t_{r}$ is the bunch return time.

It follows from Eq. (1) that the current limit due to the $n$th HOM is inversely proportional to the quantity $(R / Q)_{n} Q_{n} f_{n}$. Thus, the modes with higher shunt impedance $(R / Q)_{n}$ are considered more dangerous. In order to keep the quantity $(R / Q)_{n} Q_{n} f_{n}$ small and therefore maximize the current, $Q_{n}$ for such modes must be reduced by means of external couplers or ferrite absorbers, usually located in the beam pipe sections of the cavities [12].

For example, the highly optimized TESLA cavity (which is a $1.3 \mathrm{GHz}$ nine-cell SRF cavity) utilizes coaxial couplers located on the beam pipes on both sides of the cavity [14]. However, the TESLA cavity does not have HOM damping ability adequate for the high-current ERL projects [15]. Modifications of this design have been suggested to improve HOM damping by altering beam pipe and iris radii, somewhat sacrificing accelerating properties. Simulations confirmed the effectiveness of the new designs at providing the HOM damping required for $100 \mathrm{~mA}$ operation of the corresponding ERLs $[15,16]$.

Other examples are the five-cell cavities developed at JLab $[7,17,18]$ for the frequencies of $748.5 \mathrm{MHz}$ and $1.497 \mathrm{GHz}$. These designs are specifically optimized for HOM damping. Each design utilizes six waveguides on the beam pipe (three on each side, arranged in a $Y$ shapea so-called "waveguide end group"). Simulations [18] have demonstrated that a BBU threshold of $1 \mathrm{~A}$ can be achieved for the $1.497 \mathrm{GHz}$ cavity in a proposed ERL-based free electron laser.

Another design was recently proposed for a $1.4 \mathrm{GHz}$ nine-cell cavity with waveguides attached directly to the accelerating cells, coupling through thin slots [8]. Simulations have shown its ability to provide a BBU current threshold as high as $1.5 \mathrm{~A}$ if used for the driver accelerator in the proposed Advanced Photon Source upgrade [4]. The design can be very promising if the required confinement of the fundamental mode is demonstrated. But good HOM suppression comes at a price of increased surface fields and as a result a decreased accelerating gradient.

Photonic band gap (PBG) cells are known for good HOM suppression due to their intrinsic frequency selectivity, which can be used to confine the accelerating mode but not the HOMs $[19,20]$. A SRF PBG prototype resonator, operating at the frequency of $11 \mathrm{GHz}$, was designed and fabricated about two decades ago [21]. The resonator's $Q$ factor was shown to be at least $1.2 \times 10^{6}$ at the temperature of $4.8 \mathrm{~K}$. The first multicell PBG accelerating structure was designed and tested with an electron beam at MIT a decade ago [22]. This was a room-temperature traveling wave structure operating at the frequency of $17.14 \mathrm{GHz}$. Recently, it has been experimentally shown that a similar PBG module at 11.7 GHz has reduced HOM wakefields [23]. A dielectric PBG structure was designed and optimized for stronger HOM damping at the University of Colorado [24]. A hybrid (metal and dielectric) PBG module was recently designed and tested at high power at MIT [25].

Advances in PBG technology for room-temperature accelerators revived interest in superconducting PBG cavities. A superconducting PBG resonator design was proposed in Ref. [26]. A prototype, operating at the frequency of $16 \mathrm{GHz}$, was tested at cryogenic temperatures and showed $Q=1.2 \times 10^{5}$ limited by radiation losses. Recently, several single PBG superconducting cells operating at $2.1 \mathrm{GHz}$ were fabricated and tested at Los Alamos National Laboratory. The results showed that high accelerating gradients $(18 \mathrm{MV} / \mathrm{m}$ at the temperature of $4 \mathrm{~K})$ and high cavity $Q$ factors $\left(4 \times 10^{9}\right.$ at the temperature of $1.9 \mathrm{~K})$ could be achieved in the PBG cells $[27,28]$.

A 2D triangular lattice of conducting rods is used in both designs $[27,28]$ for the previously tested superconducting PBG cells and was adopted for the design presented in this paper. Maxwell's equations can be solved to obtain two sets of eigenmode solutions (the TM and the TE polarizations) propagating in the 2D lattice of metal rods [29]. Frequencies of those solutions, plotted as functions of the wave vector $\vec{k}$ in the transverse plane, form "bands" in the dispersion diagram (solid lines in Fig. 1). A frequency range where no solution exists for one of the polarizations is called a band gap (hence, the name PBG structures). Gap sizes and positions are functions of the ratio of the rod radius, $a$, to the spacing between the rods, $b$. For $a / b=0.15$ used in all previously tested SRF PBG cells, only one band gap exists for the TM mode polarization (yellow region in Fig. 1), and no gaps exist for the TE polarization [19].

In PBG accelerating cells, the central rod is removed from the 2D lattice of rods to form a hexagonally shaped "cavity." The choice of parameters ensures that the lowest frequency mode (monopole) in the cavity is trapped, because the TM waves cannot propagate through the lattice 


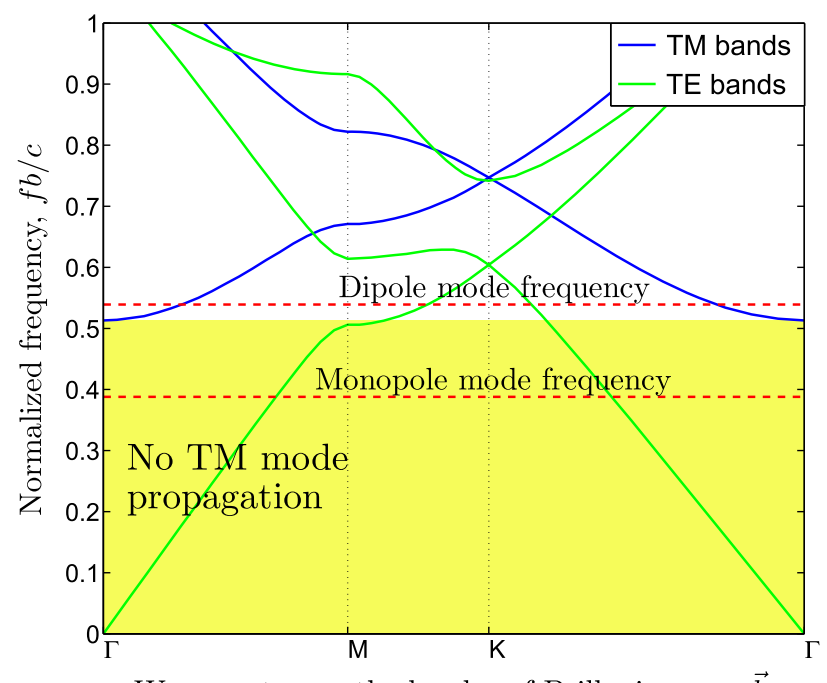

Wave vector on the border of Brillouin zone, $\vec{k}$

FIG. 1. Frequency of eigenmode solutions in an infinite photonic crystal as a function of the wave vector in the plane of the crystal. The crystal has a triangular lattice of metal rods with a ratio of the rod radius, $a$, to the spacing between the rods, $b$, equal to 0.15 . Frequencies for the monopole and the dipole modes are shown with dashed straight lines in a resonator formed by a single removed rod.

at this frequency. At the same time, the next mode (dipole) has its frequency outside of the band gap and is allowed to leak out to the periphery of the PBG structure $[19,20]$. All other HOMs have frequencies greater than that of the lowest dipole mode; thus, they propagate through the structure and can be damped (both the TM and the TE polarizations).

Designing a PBG cell for a superconducting accelerator introduces some difficulties. rfs used for superconducting accelerators are typically lower than those for roomtemperature accelerators (to reduce resistive losses [30]), which means that the accelerating cavities are bigger. Transverse size restrictions limit the structure to only two layers of PBG rods [27]. Although the fields of the accelerating mode decay exponentially with distance from the center, the two layers of rods are not sufficient to create a structure's diffraction $Q$ larger than the SRF cavity's Ohmic $Q>10^{8}$. Thus, the superconducting cell has to have an outer wall enclosing the two-layer lattice of rods.

In this paper, we present the first multicell accelerating cavity that incorporates a PBG cell similar to the single cells described above. The cavity has five cells: four elliptical cells and one PBG cell in the middle (Fig. 2). The presented design is well suited for high-current operation where strong HOM damping is required and, at the same time, has accelerating properties and surface fields similar to the more conventional cavities that use only elliptical cells. In addition, the PBG cavity does not need HOM couplers on the beam pipe, which means that it has an advantage of the increased "real estate" gradient due to

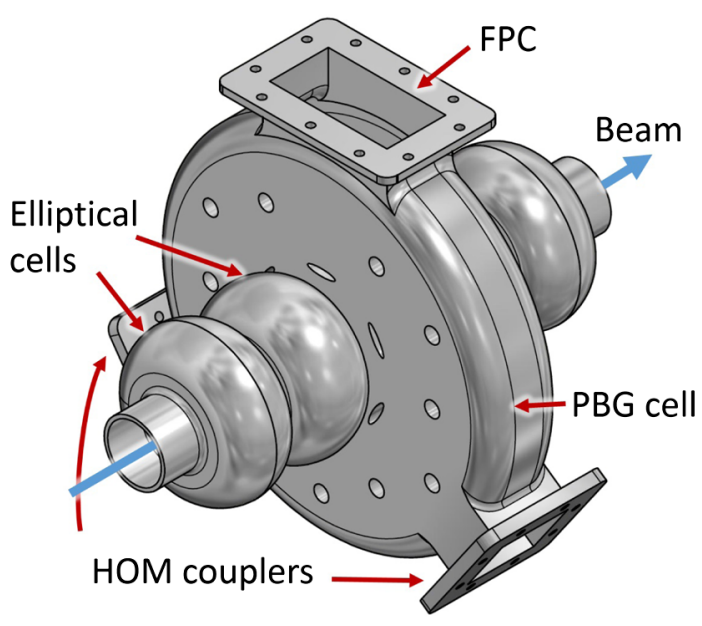

FIG. 2. Drawing of the $2.1 \mathrm{GHz}$ five-cell cavity with the PBG cell replacing one of the elliptical cells.

its shorter length. A detailed discussion of the cavity design and the accelerating properties can be found in Sec. II of this paper. HOM simulations performed with two independent approaches (wakefield and eigenmode) are discussed in Sec. III. The cavity fabrication and tuning process are described in Sec. IV. External $Q$-factor measurements that verified the simulations are described in Sec. V. Conclusions are summarized in Sec. VI.

\section{DESIGN OF THE FIVE-CELL SRF CAVITY}

The four elliptical cells in the five-cell cavity were chosen to be of the "low-loss" shape. The low-loss shape was originally developed for JLab's highly damped fivecell module at $748.5 \mathrm{MHz}$ described in Ref. [17]. The dimensions were scaled to the frequency of $2.1 \mathrm{GHz}$.

The PBG cell incorporated in the five-cell cavity is similar to the single cells described in Ref. [31]. The key difference is that the three waveguides are attached to the periphery of the cell. As shown in Figs. 2 and 3, one of the waveguides has a larger cross section (waveguide size WR430) and is used both as an HOM coupler and as the fundamental power coupler (FPC). The cavity was planned to be tested at Los Alamos National Laboratory with a CW beam current of $100 \mathrm{~mA}$ and a bunch repetition frequency of $100 \mathrm{MHz}$. This high beam current demanded strong coupling to the fundamental mode $\left(Q_{\text {ext }}=2 \times 10^{4}\right)$, so the PBG rod located closest to the FPC was removed. The coupling can be adjusted towards higher $Q_{\text {ext }}$ with a waveguide stub.

Two smaller waveguides (cutoff for the fundamental mode) were used as HOM dampers and had customized dimensions. HOM simulations described in the next section were used to optimize the two HOM couplers.

Similar to some single PBG cells tested previously [28], this design takes advantage of altering the shape of the inner layer of rods, as can be seen in Fig. 3. Going from a 


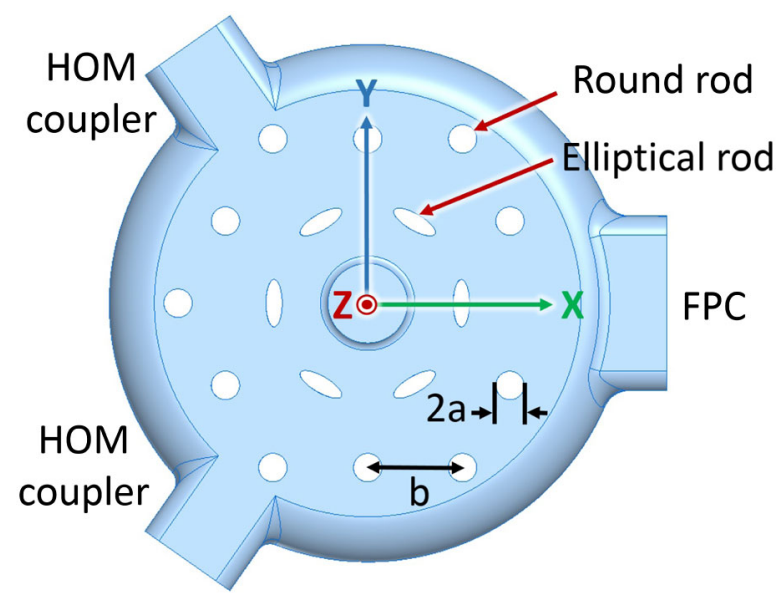

FIG. 3. PBG cell as seen from the $Z$ axis pointing along the beam trajectory. The vacuum region is shown in blue.

round to an elliptical shape lowers the surface curvature and decreases the peak surface magnetic field. Doing so does not make the HOM damping properties of the cell worse [31].

However, even with the elliptically shaped rods, the PBG cell still has a higher ratio of the peak surface magnetic field to the accelerating gradient than the elliptical cell. For that reason, the cells in the five-cell cavity were slightly detuned to obtain an uneven accelerating gradient profile $[28,32]$. This ensured that the probability of quenching in the PBG cell was no higher than in any other cells. The design accelerating gradient profile is shown in Fig. 4 with the dashed blue line.

Dimensions of the designed five-cell cavity are listed in Table I. Table II compares the main accelerating properties of the five-cell PBG cavity to the five-cell cavity with only

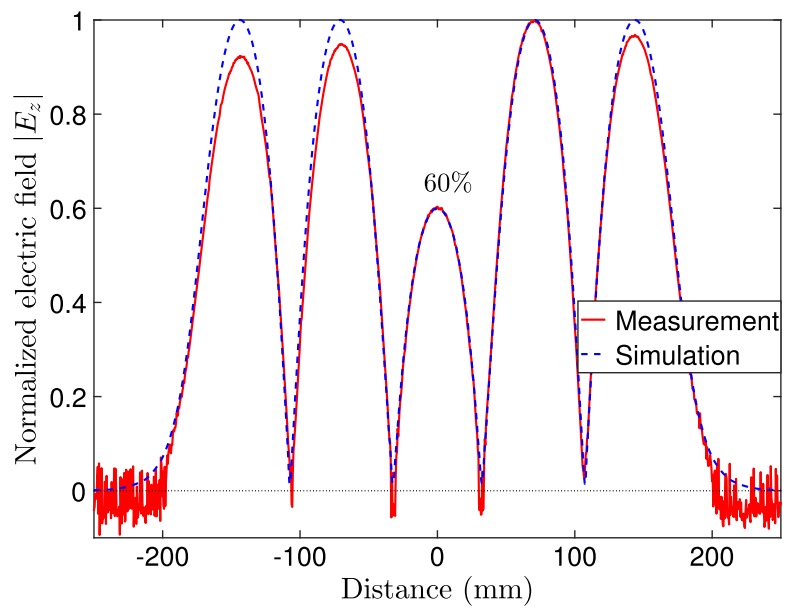

FIG. 4. The design accelerating the gradient profile, compared to the profile measured in the welded cavity using the beadpulling method. The field in the center cell is intentionally lowered to ensure an equal peak surface magnetic field in every cell.
TABLE I. Dimensions of the five-cell cavity with a PBG cell.

Cavity length $\quad 35.1 \mathrm{~cm}$

Inner beam pipe radius

Inner diameter of the elliptical cells

$2.49 \mathrm{~cm}$

Inner diameter of the PBG cell

$12.6 \mathrm{~cm}$

Outer diameter of the round PBG rods

$32.4 \mathrm{~cm}$

Major outer semiaxis of the elliptical PBG rods

$1.78 \mathrm{~cm}$

$1.49 \mathrm{~cm}$

$0.51 \mathrm{~cm}$

Minor outer semiaxis of the elliptical PBG rods

Spacing between the round rods

$5.94 \mathrm{~cm}$

Spacing between the elliptical rods

$5.87 \mathrm{~cm}$

TABLE II. Accelerating properties of the five-cell cavity with a PBG cell compared to the accelerating properties of the cavity with five elliptical low-loss cells from Ref. [17].

\begin{tabular}{lcc}
\hline \hline & PBG & $\begin{array}{c}\text { JLab's five elliptical } \\
\text { cells cavity }\end{array}$ \\
\hline Frequency $\left(f_{0}\right)$ & $2100 \mathrm{MHz}$ & $748.5 \mathrm{MHz}$ \\
Shunt impedance $\left(\frac{R}{Q}\right)$ & $515 \Omega$ & $525 \Omega$ \\
Geometrical factor $(G)$ & $265 \Omega$ & $276 \Omega$ \\
Peak surface electric field ratio & 2.65 & 2.50 \\
$\quad\left(E_{\text {peak }} / E_{\text {acc }}\right)$ & $4.48 \frac{m T}{\frac{M V}{m}}$ & $4.27 \frac{m T}{\frac{M V}{m}}$ \\
$\begin{array}{l}\text { Peak surface magnetic field } \\
\text { ratio }\left(B_{\text {peak }} / E_{\text {acc }}\right)\end{array}$ & \\
\hline \hline
\end{tabular}

elliptical cells [17]. Although a single PBG cell by itself has higher surface fields and a lower shunt impedance than an elliptical cell [32], the corresponding parameters of the designed five-cell cavity are only slightly worse. Indeed, the differences in the shunt impedance, the peak surface electric field, and the peak surface magnetic field are within a few percent, as shown in Table II. However, since there are no couplers in the beam pipes, the real estate gradient can be as much as $10 \%$ higher (the exact number depends on the cavity packing fraction).

\section{HOM SIMULATIONS}

Simulations presented in this section were used to optimize positions and dimensions of the HOM couplers and the FPC for the strongest HOM damping. A MATLAB routine was used together with electromagnetic simulations to tune individual parameters such as the positions and dimensions of the coupler waveguides and outside dimensions of the PBG cell. The simulation data presented below apply to the final optimized geometry.

We studied HOMs paying particular attention to the monopole and the dipole modes with frequencies below the corresponding beam pipe's cutoffs. Modes with higher order angular variation were not considered, because their transverse impact on the beam is smaller. Modes with frequencies above the beam pipe's cutoff were considered less dangerous, as they propagate through the beam pipe and do not build up in the cavity. 
To classify HOMs in the cavity, we used two symmetry planes: $X Y$ and $X Z$ (here $Z$ is the axis along the beam line and the $X$ axis is parallel to the FPC waveguide, as shown in Fig. 3). All the dipole modes can be classified as either $X$ modes (the $\mathbf{E}$ field is in the $X Z$ plane) or $Y$ modes (the $\mathbf{E}$ field is perpendicular to the $X Z$ plane).

We used two different approaches to study HOMs. First, wakefield simulations were performed in Computer Simulation Technology (CST) Particle Studio [33] to identify the most dangerous dipole modes and compute the corresponding deflecting voltages induced by a single bunch. Next, eigenmode simulations were performed in Ansys high frequency structural simulator (HFSS) [34] to verify the results obtained from the wakefield simulations and to calculate the external $Q$ factors for the modes. The monopole modes were also studied in the eigenmode approach.

\section{A. Wakefield simulations}

CST Particle Studio [33] was used to analyze the dipole HOMs in the designed five-cell cavity. In particular, the wakefield solver was employed to simulate interactions of the HOMs with an electron beam in the designed five-cell cavity. The wakefield solver computes the fields excited in the structure by a source charge and simulates how the fields can affect a test charge entering the cavity some time after the source charge.

We calculated the effective deflecting voltages $V_{\perp n}$ that describe how the $n$th dipole mode affects the test charge traveling offset from the axis. $V_{\perp n}$ is defined in Ref. [30] as

$$
V_{\perp n}=\frac{1}{q} \int_{0}^{L} \widetilde{F_{\perp n}} e^{i \omega_{n} z / c} d z .
$$

Here $\widetilde{F_{\perp n}}$ is a complex phasor of the transverse electromagnetic force acting on the test charge $q\left(F_{\perp n}=\right.$ $\left.\operatorname{Re}\left[\widetilde{F_{\perp n}} \exp \left(i \omega_{n} z / c\right)\right] \exp \left[-\omega_{n} z /\left(2 c Q_{n}\right)\right]\right), L$ is the length of the structure, $c$ is the speed of light, and $\omega_{n}$ and $Q_{n}$ are the angular frequency and the quality factor of the $n$th HOM, respectively.

In order to find the deflecting voltages, we first computed the wake potential and the wake impedance, defined in Ref. [35] as

$$
\begin{gathered}
\mathbf{w}_{\perp}\left(\mathbf{r}, \mathbf{r}_{1}, s\right)=\frac{1}{q q_{1}} \int_{0}^{L} d z \mathbf{F}_{\perp t=(z+s) / c}, \\
\mathbf{Z}_{\perp}\left(\mathbf{r}, \mathbf{r}_{1}, \omega\right)=\frac{1}{i c} \int_{-\infty}^{\infty} \mathbf{w}_{\perp}\left(\mathbf{r}, \mathbf{r}_{1}, s\right) e^{-i \omega s / c} d s .
\end{gathered}
$$

Here $\mathbf{r}$ is the transverse displacement of the source charge $q_{1}, \mathbf{r}_{1}$ is the transverse displacement of the test charge $q$, and $s$ is the distance between the charges. The wakefield simulations were performed for the cases $\mathbf{r}=\mathbf{r}_{1}=x \hat{x}$ and $\mathbf{r}=\mathbf{r}_{1}=y \hat{y}$. The calculated transverse wake impedance $Z_{\perp}$ for both cases ( $X$ or $Y$ displacement) is shown in Fig. 5.
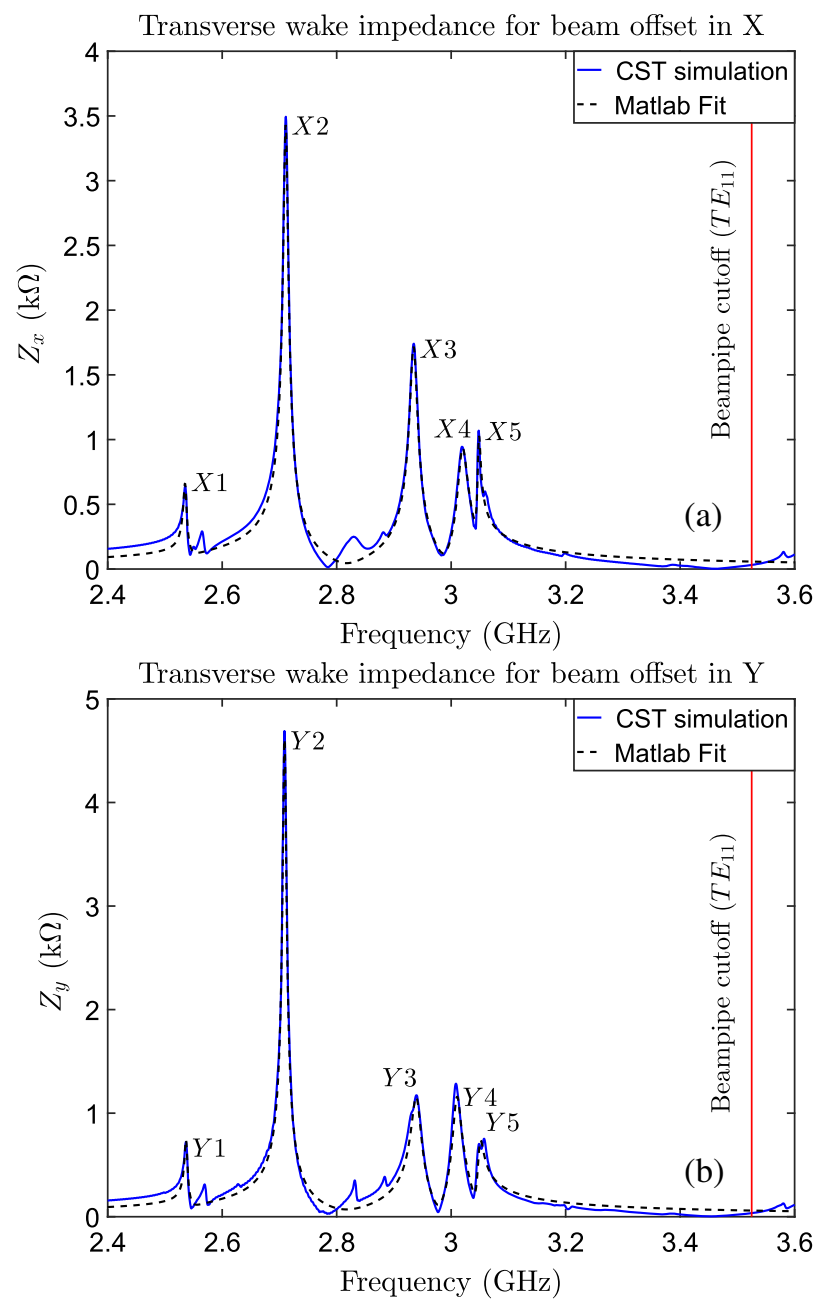

FIG. 5. Transverse impedance calculated by CST Particle Studio for the electron beam displaced in the $X$ direction (a) and the $Y$ direction (b) by $6.35 \mathrm{~mm}$. The ten most dangerous modes correspond to the biggest peaks in impedance.

As demonstrated in the Appendix, the deflecting voltage for a dipole mode is proportional to the integrated area under the corresponding peak of the transverse wake impedance. Since we are interested only in the deflecting voltages, the energy decay time can be chosen independently, as it affects only the width of a peak but not the area. As long as the wake impedance peaks do not overlap, we can save computational time by decreasing the energy decay time and widening the peaks. For that reason, a lossy material was used for the cavity walls in addition to the perfectly matched layer (PML) [36] that covered the waveguide couplers.

In Fig. 5, ten peaks can be seen in wake simulations that correspond to the ten most dangerous dipole modes with the highest $V_{\perp}$. These modes were labeled as $X_{1}-X_{5}$ and $Y_{1}-Y_{5}$ and were used for the cavity optimization. Deflecting voltages were calculated by fitting the known analytical shapes for wake impedance peaks (see the Appendix) with variable complex phases. The calculated 
TABLE III. Simulation results for the dipole modes with $(R / Q)^{d}>1 \Omega$.

\begin{tabular}{|c|c|c|c|c|}
\hline$f(\mathrm{GHz})$ & $Q_{\text {ext }}$ & $(R / Q)^{d}(\Omega)$ & $\begin{array}{c}\left|V_{\perp}\right| /(q \rho)[\mathrm{V} /(\mathrm{nC} \mathrm{mm})] \\
\text { (eigenmode) }\end{array}$ & $\begin{array}{c}\left|V_{\perp}\right| /(q \rho)[\mathrm{V} /(\mathrm{nC} \mathrm{mm})] \\
\text { (wakefield) }\end{array}$ \\
\hline \multicolumn{5}{|l|}{$X$ modes } \\
\hline $2.538\left(X_{1}\right)$ & $2.7 \times 10^{3}$ & 7.7 & 3.2 & 3.2 \\
\hline 2.567 & $1.5 \times 10^{3}$ & 3.6 & 1.6 & \\
\hline $2.713\left(X_{2}\right)$ & $7.5 \times 10^{2}$ & 58.4 & 28.3 & 29.4 \\
\hline 2.713 & $1.3 \times 10^{2}$ & 9.3 & 4.5 & \\
\hline 2.831 & $1.0 \times 10^{2}$ & 8.5 & 4.5 & \\
\hline 2.884 & $6.8 \times 10^{2}$ & 1.1 & 0.6 & \\
\hline $2.936\left(X_{3}\right)$ & $3.0 \times 10^{2}$ & 42.1 & 23.8 & 22.7 \\
\hline 2.962 & $1.8 \times 10^{2}$ & 2.1 & 1.2 & \\
\hline $3.020\left(X_{4}\right)$ & $2.0 \times 10^{2}$ & 27.6 & 16.6 & 15.0 \\
\hline $3.047\left(X_{5}\right)$ & $1.6 \times 10^{4}$ & 5.7 & 3.5 & 4.1 \\
\hline 3.060 & $4.8 \times 10^{2}$ & 2.4 & 1.5 & \\
\hline \multicolumn{5}{|l|}{$Y$ modes } \\
\hline $2.539\left(Y_{1}\right)$ & $2.7 \times 10^{3}$ & 8.8 & 3.7 & 3.3 \\
\hline 2.571 & $2.7 \times 10^{4}$ & 3.0 & 1.3 & \\
\hline 2.710 & $5.4 \times 10^{2}$ & 8.9 & 4.3 & \\
\hline $2.711\left(Y_{2}\right)$ & $9.4 \times 10^{2}$ & 57.7 & 27.9 & 29.2 \\
\hline 2.825 & $3.7 \times 10^{1}$ & 7.2 & 3.8 & \\
\hline 2.887 & $5.4 \times 10^{3}$ & 1.3 & 0.7 & \\
\hline $2.940\left(Y_{3}\right)$ & $2.6 \times 10^{2}$ & 35.3 & 20.1 & 20.1 \\
\hline 2.995 & $1.4 \times 10^{2}$ & 3.0 & 1.7 & \\
\hline $3.014\left(Y_{4}\right)$ & $1.9 \times 10^{2}$ & 26.6 & 15.9 & 17.7 \\
\hline $3.048\left(Y_{5}\right)$ & $2.2 \times 10^{3}$ & 5.8 & 3.6 & 6.1 \\
\hline 3.059 & $7.3 \times 10^{2}$ & 3.4 & 2.1 & \\
\hline
\end{tabular}

voltages, normalized over the source charge $q$ and the offset $\rho$, are listed in the last column of Table III.

\section{B. Eigenmode simulations}

We simulated HOMs with the eigenmode solver of Ansys HFSS. Similar to the wakefield approach, the eigenmode approach can be used to calculate voltages induced by a single bunch for different modes [30]:

$$
\frac{\left|V_{\|_{n}}\right|}{q}=\pi f_{n}\left(\frac{R}{Q}\right)_{n}
$$

for the $n$th monopole mode and

$$
\frac{\left|V_{\perp n}\right|}{q \rho}=\frac{2 \pi^{2} f_{n}^{2}}{c}\left(\frac{R}{Q}\right)_{n}^{d}
$$

for the $n$th dipole mode. Here $f_{n}$ is the frequency of the mode, $q$ is the charge of the exciting bunch, $\rho$ is the offset from the axis, $(R / Q)_{n}$ is the shunt impedance of a monopole mode, and $(R / Q)_{n}^{d}$ is the shunt impedance of a dipole mode. Both monopole and dipole shunt impedances are measured in ohms in agreement with Ref. [30].

For each HOM, an eigenmode simulation was set up to calculate the shunt impedance and the external $Q$ factor. The complex geometry of the PBG cell supports a large number of unimportant modes trapped in the lattice of rods with very small fields at the center of the cell. While not interacting strongly with the beam, those modes can unnecessarily increase the computational time. For that reason, only modes with $R / Q>1 \Omega$ (for both monopoles and dipoles) were studied in detail.

In order to calculate a dipole mode's shunt impedance $(R / Q)^{d}$, the beam trajectory was shifted off axis in either the $X$ or the $Y$ direction by a distance equal to one beam pipe's radius. To calculate the external quality factors, all three waveguides were covered with a PML. The calculated shunt impedances and $Q_{\text {ext }}$ for the dipole and the monopole modes (with shunt impedance exceeding $1 \Omega$ ) are presented in Fig. 6 and Tables III and IV. Tables III and IV also list the estimated voltages induced by a single bunch and compare them to the voltages obtained in the wakefield simulations for the ten dangerous modes. Good agreement between the two independent approaches shows the reliability of the obtained results.

We were particularly interested in the $Q_{\text {ext }}$ for the dipole modes as they are the major cause of the BBU instability. As can be seen in Table III, most of the dipole modes have $Q_{\text {ext }}$ in the range of $10^{2}-10^{3}$. Only two modes $(f=3.047 \mathrm{GHz}$ and $f=2.571 \mathrm{GHz})$ with relatively low shunt impedances of $5.7 \Omega$ and $3.0 \Omega$ have higher $Q_{\text {ext }}: 1.6 \times 10^{4}$ and $2.7 \times 10^{4}$, respectively. This can be explained by the fact that the longitudinal component of the 

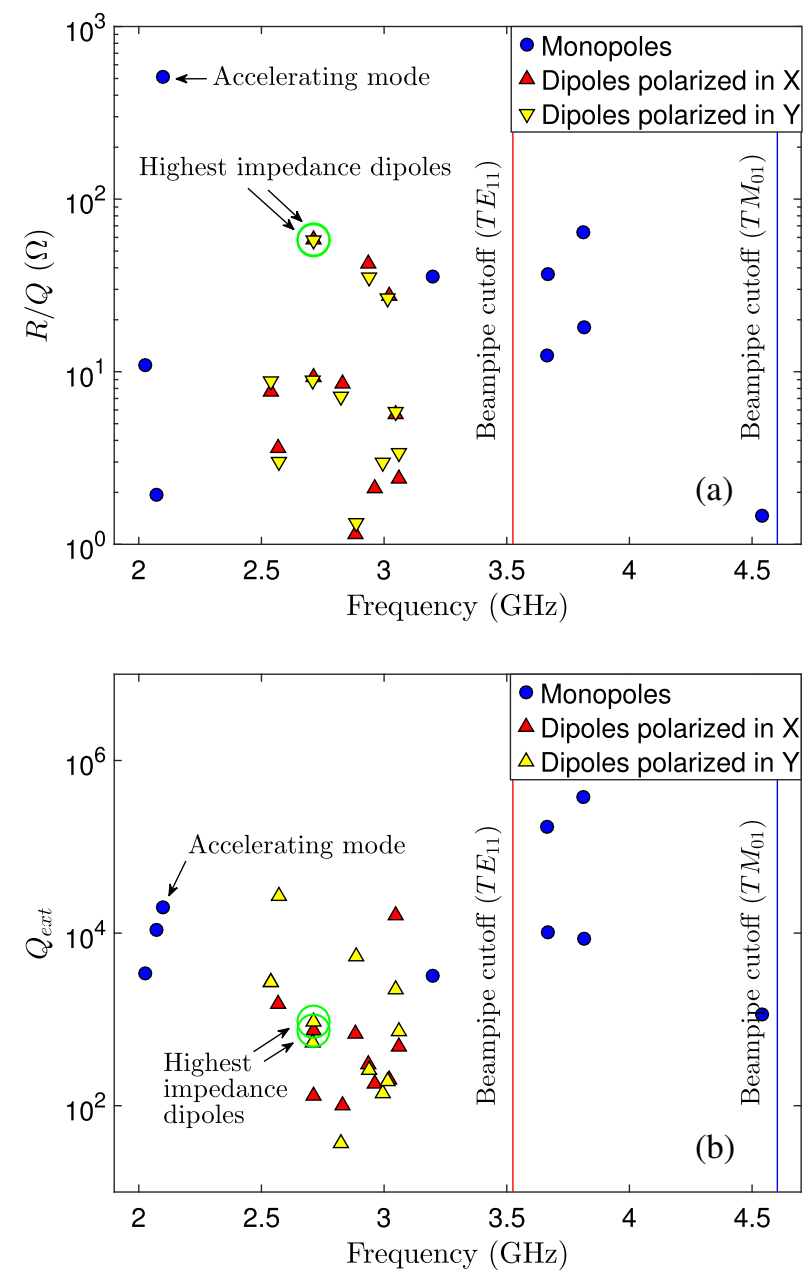

FIG. 6. Shunt impedances (a) and external $Q$ factors (b) for monopoles and dipoles in the five-cell PBG cavity (simulation). Only modes with $R / Q \geq 1 \Omega$ are shown.

electric field $E_{z}$ for these two modes is going through zero in the middle of the PBG cell. Although some asymmetry due to fabrication errors should lower the $Q_{\text {ext }}$ for such modes, asymmetrical tuning could be used to bring $Q_{\text {ext }}$ even lower, if necessary.

TABLE IV. Simulation results for the monopole modes with $R / Q>1 \Omega$.

\begin{tabular}{lccc}
\hline \hline Freq $(\mathrm{GHz})$ & $Q_{\mathrm{ext}}$ & $R / Q(\Omega)$ & $\left|V_{\|_{n}}\right| / q(\mathrm{~V} / \mathrm{nC})$ \\
\hline $2.025(\pi / 5)$ & $3.4 \times 10^{3}$ & 10.9 & 69.3 \\
$2.071(3 \pi / 5)$ & $1.1 \times 10^{4}$ & 1.9 & 12.7 \\
$2.100(\pi)$ & $2.0 \times 10^{4}$ & 515 & 3400 \\
3.198 & $3.2 \times 10^{3}$ & 35.7 & 359.1 \\
3.663 & $1.7 \times 10^{5}$ & 12.5 & 143.5 \\
3.668 & $1.0 \times 10^{4}$ & 36.6 & 421.6 \\
3.813 & $3.8 \times 10^{5}$ & 64.5 & 773.1 \\
3.814 & $8.6 \times 10^{3}$ & 18.2 & 217.8 \\
4.540 & $1.1 \times 10^{3}$ & 1.5 & 20.9 \\
\hline \hline
\end{tabular}

TABLE V. Comparison between simulated and measured external $Q$ factors for six of the dangerous HOMs.

\begin{tabular}{lccc}
\hline \hline Mode & $f(\mathrm{GHz})$ & $Q_{\text {ext }}$, HFSS & $Q_{\text {ext }}$, measured \\
\hline$X_{1}$ & 2.538 & $2.7 \times 10^{3}$ & $2.7 \times 10^{3}$ \\
$X_{2}$ & 2.713 & $7.5 \times 10^{2}$ & $9.4 \times 10^{2}$ \\
$X_{3}$ & 2.936 & $3.0 \times 10^{2}$ & $1.4 \times 10^{2}$ \\
$Y_{1}$ & 2.539 & $2.7 \times 10^{3}$ & $3.0 \times 10^{3}$ \\
$Y_{2}$ & 2.711 & $9.4 \times 10^{2}$ & $1.3 \times 10^{3}$ \\
$Y_{3}$ & 2.940 & $2.6 \times 10^{2}$ & $1.1 \times 10^{2}$ \\
\hline \hline
\end{tabular}

The BBU figure of merit $(R / Q)_{n} Q_{n} f_{n}$ is plotted in Fig. 7 for the PBG cavity, as well as for the nine-cell TESLA cavity which employs coaxial HOM couplers [14,37]. Loaded $Q$ factors were substituted with the calculated values of $Q_{\text {ext }}$ for all of the plotted modes due to negligible losses in superconducting walls. It can be seen that the highest value of $(R / Q)_{n} Q_{n} f_{n}$ for the dipole modes below a beam pipe cutoff is much lower for the PBG cavity than for the TESLA cavity. This means that the PBG cavity has the potential to support much higher beam currents due to improved HOM damping.

\section{FABRICATION AND TUNING}

The prototype cavity was fabricated by Niowave, Inc., from a combination of fine-grain niobium sheets and machined ingot parts, joined by electron-beam welding.

First, halves of the elliptical cells were fabricated. The halves were welded together at the irises prior to welding them to the PBG cell to minimize the effect of weld shrinkage on field flatness. Then, two halves of the PBG cell were stamped, fitted, and joined with the PBG rods.

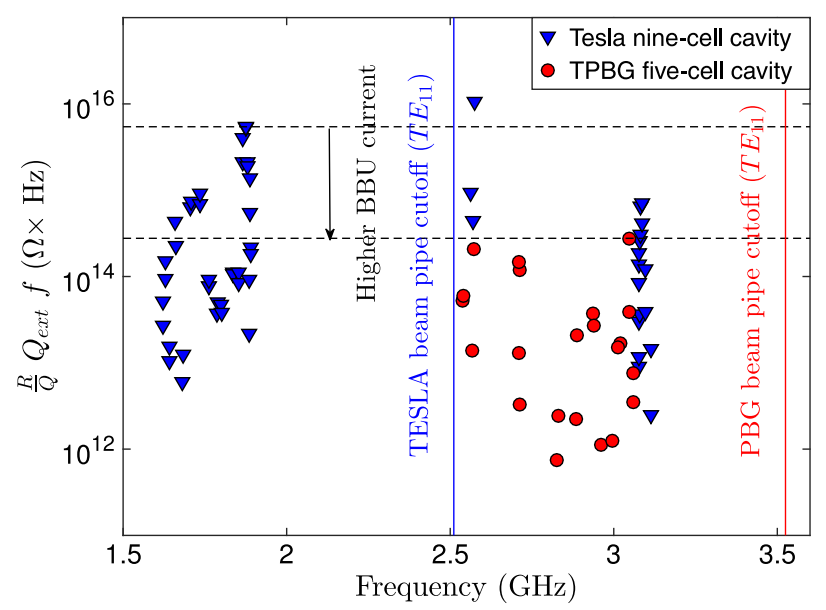

FIG. 7. BBU figure of merit $(R / Q) Q_{\text {ext }} f$ for the dipole modes for two different cavities: the nine-cell TESLA and the five-cell PBG. Dipole shunt impedance is in units of ohms. The dashed lines mark the highest values of the figure of merit for HOMs with frequencies below the corresponding beam pipe cutoffs. Data for the TESLA cavity are from Refs. [14,37]. 

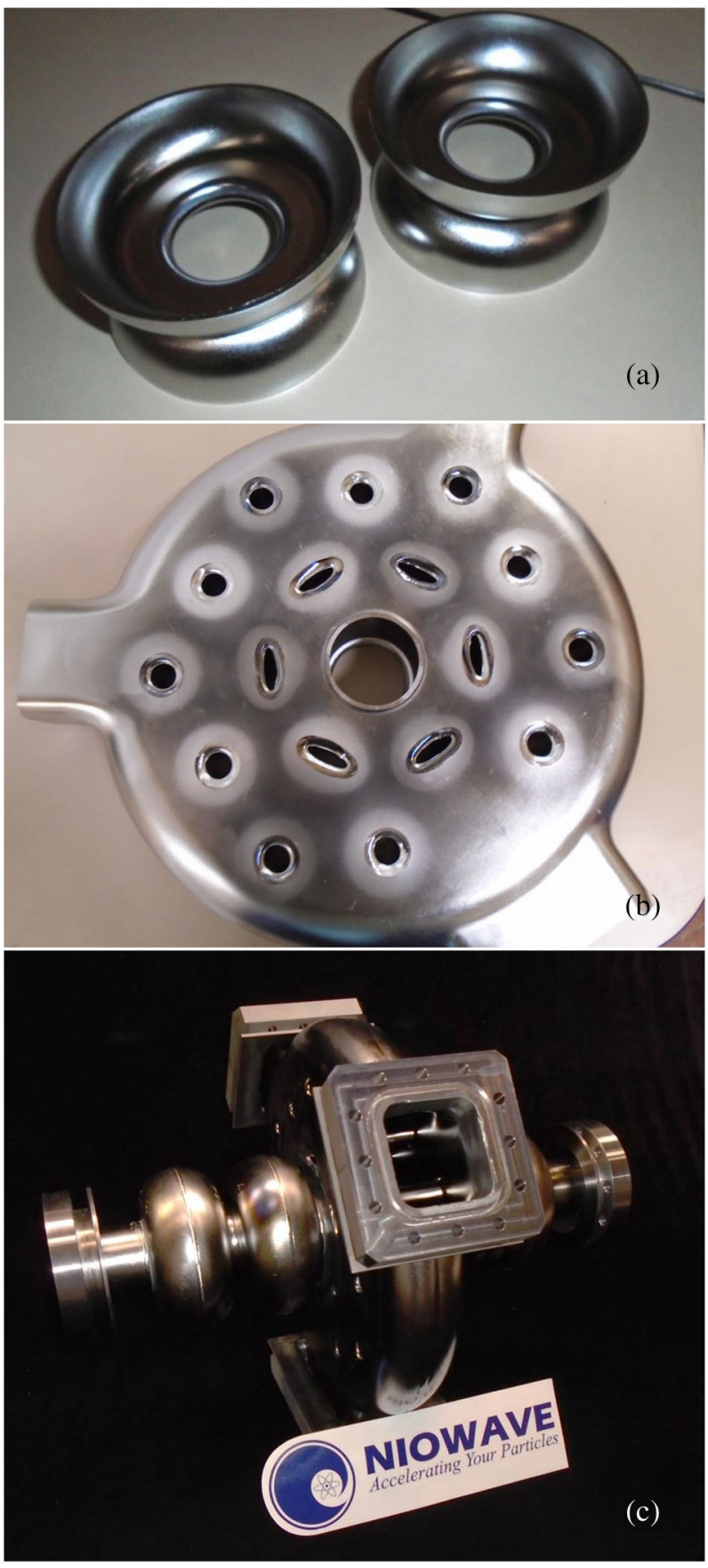

FIG. 8. Halves of the elliptical cells after being welded together at the irises (a). Photo of the PBG cell with welded rods (b). Final photo of the fabricated five-cell cavity (c).

Finally, all five cells were welded together in several steps with tuning done between the welding steps. Figure 8 shows photographs of parts of the cavity taken during fabrication, together with a photograph of the final cavity.

After welding, the cavity underwent a surface treatment. It was chemically treated using $1: 1: 2$ buffered chemical polish solution of $\mathrm{HF}, \mathrm{HNO}_{3}$, and $\mathrm{H}_{3} \mathrm{PO}_{4}$ to etch $150(\mu) \mathrm{m}$ of the inner niobium surface and high-pressure rinsed with ultrapure water.
The cavity's $Q$ factor measured at room temperature was $Q_{300 \mathrm{~K}}=7300$, indicating a bulk conductivity of $\left(\sigma=6.3 \times 10^{6} \Omega^{-1} \mathrm{~m}^{-1}\right)$, in good agreement with the literature value for niobium at room temperature of $6.2 \times 10^{6} \Omega^{-1} \mathrm{~m}^{-1}$ [38].

Tuning was done between the welding steps by trimming halves of each elliptical cell at the equator. The gradient profile after the final weld was within $5 \%$ of the design without additional tuning. Slight mechanical adjustments to the elliptical cells postweld produced the final field profile shown as a solid red line in Fig. 4.

For the purpose of this particular testing, it was not necessary to tune the cavity to exactly $2.100 \mathrm{GHz}$; therefore, the center (PBG) cell was not tuned. Nevertheless, the resulting frequency of the cavity $(2.1062 \mathrm{GHz}$ in liquid helium, as measured in high-gradient tests $[39,40]$ ) was within $0.5 \%$ of the design frequency.

In a multicavity linac, all the structures must be tuned to the same frequency. Therefore, a few different mechanisms of tuning have been proposed, including pushing on the inside of the PBG rods. A cavity tuner which stresses and compresses the full cavity to tune the frequency of the structure will affect the field flatness, because the elliptical cells will tune more easily than the center PBG cell. Nevertheless, we expect such a scheme to allow sufficient tuning to synchronize a multicavity linac with little cost in terms of field flatness.

\section{HOM MEASUREMENTS}

The fabricated cavity was used to measure the HOM damping properties. To obtain $Q_{\text {ext }}$ of the HOMs, we separately measured the values of loaded $Q$ factors $Q_{L}$ and the unloaded $Q$ factors $Q_{0}$ and used the following relationship:

$$
\frac{1}{Q_{\mathrm{ext}}}=\frac{1}{Q_{L}}-\frac{1}{Q_{0}}
$$

For SRF cavities, a cryogenic test is usually necessary to accurately measure $Q_{\text {ext }}$ values if they are much greater than the unloaded $Q$ factors $Q_{0}$ at room temperature. In this case, according to Eq. (8), a change in $Q_{\text {ext }}$ only slightly affects the measured values of the total $Q$ factors $Q_{L}$ that are dominated by the Ohmic losses. However, in our case, all of the dangerous dipole modes listed in Table III are damped to $Q_{\text {ext }}$ lower than or comparable to their roomtemperature $Q_{0}$ (typically around $5 \times 10^{3}$ in the niobium cavity). Therefore, it was sufficient to do a series of much simpler room-temperature tests to characterize $\mathrm{HOM}$ damping as, for example, in Ref. [17].

The HOMs were excited with two weakly coupled loop antennas inserted through the beam pipes. The strength of coupling could be varied by adjusting the depth of the antenna insertion. Weak coupling was used to not affect the measurements of the $Q$ factors associated with the 


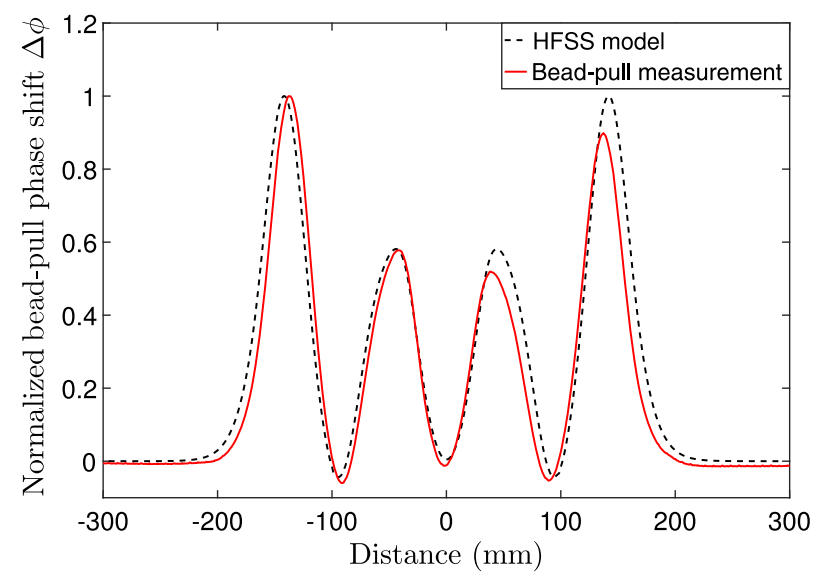

FIG. 9. Normalized phase shift in the $S_{21}$ signal obtained during the bead pull at the frequency corresponding to the $X_{2}$ mode. For comparison, the phase shift simulated in HFSS is shown.

waveguide damping. The antennas were rotated around the cavity's central axis in order to excite only one polarization of each dipole mode at a time $(X$ or $Y)$. It is worth noting that the polarizations of the $X_{1}$ and $Y_{1}$ modes were rotated relative to the $X$ and $Y$ axis, possibly due to the fabrication errors.

A bead-pull setup was incorporated in the test stand (the bead-pull technique is described in Refs. [41,42]). The setup consisted of a spherical metal bead $3.2 \mathrm{~mm}$ in diameter on a thin polyester thread, pulled by a stepper motor. The motor was controlled by a step-function generator to precisely map the location of the bead. The polyester thread was shifted off axis by about $1 \mathrm{~cm}$ to make room for the loop antennas that were exactly aligned with the cavity's central axis.

The cavity's HOM waveguide ports were tapered out to match the dimensions of the standard waveguide WR510. The farther ends of the tapers were either shorted with metal plates to measure $Q_{0}$ or terminated with rf loads to measure $Q_{L}$. The FPC was already made with the dimensions of a standard waveguide WR430 and did not need to be tapered out. Similar to the HOM waveguides, the FPC was either shorted or connected to a rf load.

First, we identified the dangerous HOMs from Table III by obtaining the field patterns corresponding to different $S_{21}$ peaks. To do that, we used the bead-pull setup to measure phase deviation of the signal transmitted between the two antennas. The phase deviation is proportional to the following combination of fields: $\epsilon E^{2}-(1 / 2) \mu H^{2}$ [42]. As an example, the phase deviation profile obtained in a beadpull measurement for the $X_{2}$ mode is shown in Fig. 9. It agrees with the normalized theoretical prediction for the phase deviation obtained in HFSS, also shown in Fig. 9. Hence, we were able to identify and separate the dangerous modes listed in Table III despite mode overlapping.

Second, $Q_{0}$ and $Q_{L}$ of the dangerous modes were obtained by measuring the widths of the corresponding
$S_{21}$ peaks with the appropriate waveguide boundary conditions. The obtained values of $Q_{0}$ and $Q_{L}$ were plugged into Eq. (8) to yield $Q_{\text {ext }}$. This method works well for $Q_{\text {ext }} \gg 10^{2}$, when the frequency and field distribution are not very sensitive to the positions of the shorts in the waveguides.

Quality factors for six dangerous dipole HOMs below $3 \mathrm{GHz}$ were measured. The results are summarized in Table V. For all the measured modes except the modes $X_{3}$ and $Y_{3}$, we could see a good agreement between the simulations and the measurements. The modes $X_{3}$ and $Y_{3}$ show $Q_{\text {ext }}$ that are even lower than the expected values.

We estimated errors in the $Q$-factor measurements due to peak overlapping. Overlapping introduces asymmetry to a peak in $S_{21}$ and makes the left side slope different from the right side slope. We estimated the errors by comparing $Q$ factors computed separately for the left and the right sides of the $S_{21}$ peaks. The resulting errors are within $\pm 12 \%$ for all the modes and cannot explain the measured $Q$ values for the modes $X_{3}$ and $Y_{3}$. Extremely low $Q_{\text {ext }}$ of $X_{3}$ and $Y_{3}$ make them sensitive to the boundary conditions in the waveguides and the shape of the periphery of the PBG cell. It is therefore possible that the field patterns of the modes in the fabricated cavity were slightly different from those predicted in the simulations, which resulted in lower values of $Q_{\text {ext }}$.

\section{CONCLUSIONS}

We have designed and built the first multicell superconducting cavity that uses a PBG cell for the HOM damping. The HOM spectrum has been carefully analyzed using eigenmode and wakefield simulations with good agreement between the two methods. Most of the dangerous HOMs are damped to fairly low external $Q$ factors on the order of $10^{3}$.

External $Q$ factors for six dangerous HOMs were experimentally measured for the fabricated cavity. The measurements agreed with the simulations. In comparison with the TESLA nine-cell cavity, the PBG module should have a much higher beam breakup current threshold.

Effective HOM damping makes the cavity an attractive candidate for future high-current accelerators. The cavity is proposed as a harmonic linac operating at a multiple of the main frequency in Brookhaven National Laboratory's eRHIC ERL [43], which may be used to undo nonlinear distortion of the beam's longitudinal phase space induced by the main linac's waveform.

The cavity was also tested at superconducting temperatures at high accelerating gradients. The results are described in Refs. [39,40].

\section{ACKNOWLEDGMENTS}

This work was supported by DOE Office of Nuclear Physics SBIR Grant No. DE-SC0009523 the U.S. 
Department of Energy (DOE) Office of Science Early Career Research Program, and the DOE Office of High Energy Physics Grant No. DE-SC0010075.

\section{APPENDIX: RELATIONSHIP BETWEEN DEFLECTING VOLTAGES AND WAKE IMPEDANCE}

In this Appendix, we find the relationship between the effective deflecting voltages of the dipole HOMs and the sizes of the corresponding peaks in the transverse wake impedance (as plotted in Fig. 5). This relationship, stated later in Eq. (A7), follows from the analytical expression for the wake impedance peaks [Eq. (A5)]. An expression similar to Eq. (A5) has been previously derived for the longitudinal impedance using $k$-loss factors in Ref. [44].

Transverse wake potential from a relativistic point charge and related transverse wake impedance are defined in Eqs. (4) and (5). In the following derivation, we consider only the special case $\mathbf{r}=\mathbf{r}_{1}$ used in the Wakefield solver of CST Particle Studio. We also consider a component of the wake potential along a particular axis $X$ and set $\mathbf{r}=\mathbf{r}_{1}=x \hat{x}$; however, the same applies to the component along the orthogonal axis $Y$. We demonstrate that the wakefield impedance curve can be used to calculate the effective deflecting voltage $V_{x n}$ defined in Eq. (3).

The expressions for the wake potential (4) and the deflecting voltage (3) for the case $\mathbf{r}=\mathbf{r}_{1}=x \hat{x}$ become

$$
\begin{aligned}
w_{x}(x, s) & =-\frac{1}{q q_{1}} \int_{0}^{L} d z F_{x, t=(z+s) / c}, \\
V_{x n} & =\frac{1}{q} \int_{0}^{L} \widetilde{F}_{x n} e^{i \omega_{n} z / c} d z .
\end{aligned}
$$

To relate Eq. (A1) to Eq. (A2), we write the electromagnetic force in the phasor notation:

$$
F_{x n}(x, z, t)=\operatorname{Re}\left(\widetilde{F_{x n}}(x, z) e^{i \omega_{n} t}\right) e^{-\frac{\omega_{n} t}{2 Q_{n}}},
$$

where $Q_{n}$ is the total quality factor of the $n$th HOM. By plugging Eq. (A3) into Eq. (A1) and using Eq. (A2), we get

$$
w_{x}(x, s)=\frac{1}{q_{1}} \sum_{n} \operatorname{Re}\left(V_{x n} e^{i \omega_{n} s / c}\right) e^{-\frac{\omega_{n} s}{2 Q_{n} c}} .
$$

Subsequently, after integration the expression for the wake impedance (5) becomes

$$
\begin{aligned}
Z_{x}(x, \omega>0) & =\frac{1}{i c} \int_{-\infty}^{\infty} w_{x}(x, s) e^{-i \omega s / c} d s \\
& =\sum_{n} \frac{V_{x n}}{2 i q_{1}} \frac{1}{\omega_{n} /\left(2 Q_{n}\right)-i\left(\omega_{n}-\omega\right)},
\end{aligned}
$$

where the wake impedance is written as a sum of individual peaks. The absolute value of the wake impedance in the vicinity of the $n$th peak becomes

$$
\begin{aligned}
\left|Z_{x n}\right| & =\frac{V_{x n}}{2 q_{1}} \frac{1}{\sqrt{\left(\omega_{n}-\omega\right)^{2}+\omega_{n}^{2} /\left(4 Q_{n}^{2}\right)}} \\
& =\frac{V_{x n}}{4 \pi q_{1}} \frac{1}{\sqrt{\left(f_{n}-f\right)^{2}+f_{n}^{2} /\left(4 Q_{n}^{2}\right)}} .
\end{aligned}
$$

Given Eq. (A6), we find the half-max width of the $n$th peak $\Delta f_{n}=\sqrt{3} f_{n} / Q_{n}$; therefore, $f_{n}^{2} /\left(4 Q_{n}^{2}\right)=\Delta f_{n}^{2} / 12$. Thus, we can finally express the effective deflecting voltage through the height of the peak $\left|Z_{x n}^{\text {peak }}\right|=\left|Z_{x n}\right|_{f=f_{n}}$ and the width of the peak $\Delta f_{n}$ :

$$
\left|V_{x n}\right|=\frac{2 \pi}{\sqrt{3}} q_{1}\left|Z_{x n}^{\text {peak }}\right| \Delta f_{n}
$$

We can use the relation in Eq. (A7) to compute the deflecting voltages for the dipole HOMs by only using a wakefield simulation. Then, eigenmode simulations can be used to verify the results.

[1] M. W. Poole et al., in Proceedings of the 2003 Particle Accelerator Conference, Portland, OR (IEEE, New York, 2003), p. 189.

[2] T. Kasuga et al., in Proceedings of the 22nd Particle Accelerator Conference, PAC-2007, Albuquerque, NM, paper TUPMN044 (IEEE, New York, 2007), p. 1016.

[3] G. H. Hoffstaetter et al., in Proceedings of the 22nd Particle Accelerator Conference, PAC-2007, Albuquerque, NM, paper MOOBAB02 (IEEE, New York, 2007), p. 107.

[4] M. Borland, G. Decker, A. Nassiri, and M. White, in Proceedings of the 22nd Particle Accelerator Conference, PAC-2007, Albuquerque, NM, paper TUPMN089 (IEEE, New York, 2007), p. 1121.

[5] E. C. Aschenauer et al., eRHIC Design Study: An Electron-Ion Collider at BNL, arXiv:1409.1633.

[6] G. H. Hoffstaetter and I. V. Bazarov, Beam-breakup instability theory for energy recovery linacs, Phys. Rev. ST Accel. Beams 7, 054401 (2004).

[7] R. A. Rimmer et al., in Proceedings of the 22nd Particle Accelerator Conference, PAC-2007, Albuquerque, NM, paper WEPMS068 (IEEE, New York, 2007), p. 2493.

[8] Z. Liu and A. Nassiri, Novel superconducting rf structure for ampere-class beam current for multi-GeV energy recovery linacs, Phys. Rev. ST Accel. Beams 13, 012001 (2010).

[9] F. Marhauser et al., in Proceedings of the 11th European Particle Accelerator Conference, Genoa, 2008, paper MOPP140 (EPS-AG, Genoa, Italy, 2008), p. 886.

[10] R. Calaga et al., in Proceedings of the 9th European Particle Accelerator Conference, Lucerne, 2004, paper TUPKF078 (EPS-AG, Lucerne, 2004), p. 1227.

[11] V. N. Litvinenko et al., in Proceedings of the 21st Particle Accelerator Conference, Knoxville, TN, 2005, paper RPPT032 (IEEE, Piscataway, NJ, 2005), p. 2242. 
[12] S. Belomestnykh, Superconducting radio-frequency systems for high- $\beta$ particle accelerators, Rev. Accel. Sci. Techol. 05, 147 (2012).

[13] G. H. Hoffstaetter, I. V. Bazarov, and C. Song, Recirculating beam-breakup thresholds for polarized higher-order modes with optical coupling, Phys. Rev. ST Accel. Beams 10, 044401 (2007).

[14] TESLA Test Facility Linac Design report, edited by D. A. Edwards, DESY, 1995.

[15] H. Sakai, K. Shinoe, T. Furuya, S. Sakanaka, T. Suwada, T. Takahashi, K. Umemori, and M. Sawamura, Development of a $1.3 \mathrm{GHz}$ 9-cell superconducting cavity for the energy recovery LINAC, in 41st Advanced ICFA Beam Dynamics Workshop on Energy Recovery Linacs (ERL07), Daresbury, UK (CERN, Geveva, 2007), p. 56, https://accelconf .web.cern.ch/accelconf/erl07/papers/34.pdf.

[16] M. Liepe, Conceptual layout of the cavity string of the cornell erl main LINAC cryomodule, in Proceedings of the 11th Workshop on RF Superconductivity (SRF2003), Lubeck/Travemunde, Germany, paper MoP33 (KEK, Tsukuba, 2003), http://epaper.kek.jp/SRF2003/papers/ mop33.pdf.

[17] H. Wang, R. Rimmer, and F. Marhauser, in Proceedings of the 22nd Particle Accelerator Conference, PAC-2007, Albuquerque, NM, paper WEPMS070 (IEEE, New York, 2007), p. 2496.

[18] F. Marhauser, W. Clemens, G. Cheng, G. Ciovati, E. F. Daly, D. Forehand, J. Henry, P. Kneisel, S. Manning, R. Manus, R. A. Rimmer, C. Tennant, and H. Wang, in Proceedings of the 11th European Particle Accelerator Conference, Genoa, 2008, paper MOPP140 (EPS-AG, Genoa, Italy, 2008), p. 886.

[19] E. I. Smirnova, C. Chen, M. A. Shapiro, J. R. Sirigiri, and R. J. Temkin, Simulation of photonic band gaps in metal rod lattices for microwave applications, J. Appl. Phys. 91, 960 (2002).

[20] E. I. Smirnova, I. Mastovsky, M. A. Shapiro, R. J. Temkin, L. M. Earley, and R. L. Edwards, Fabrication and cold test of photonic band gap resonators and accelerator structures, Phys. Rev. ST Accel. Beams 8, 091302 (2005).

[21] D. R. Smith, D. Li, D. C. Vier, N. Kroll, and S. Schultz, Recent progress on photonic band gap accelerator cavities, AIP Conf. Proc. 398, 518 (1997).

[22] E. I. Smirnova, A. S. Kesar, I. Mastovsky, M. A. Shapiro, and R. J. Temkin, Demonstration of a 17-GHz, HighGradient Accelerator with a Photonic-Band-Gap Structure, Phys. Rev. Lett. 95, 074801 (2005).

[23] E. I. Simakov, S. A. Arsenyev, C. E. Buechler, R. L. Edwards, W. P. Romero, M. Conde, G. Ha, J. G. Power, E. E. Wisniewski, and C. Jing, Observation of Wakefield Suppression in a Photonic-Band-Gap Accelerator Structure, Phys. Rev. Lett. 116, 064801 (2016).

[24] C. A. Bauer, G. R. Werner, and J. R. Cary, Truncated photonic crystal cavities with optimized mode confinement, J. Appl. Phys. 104, 053107 (2008).

[25] J. Zhang, A. M. Cook, B. J. Munroe, M. A. Shapiro, R. J. Temkin, and H. Xu, Design and high-power testing of a hybrid photonic band-gap (PBG) accelerator structure at 17 GHz, in Proceedings of IPAC 2015 in Richmond, VA,
USA, paper WEPWA062 (JLAB, Newport News, 2015), p. 2646, https://jacowfs.jlab.org/conf/proceedings/ IPAC2015/papers/wepwa062.pdf.

[26] M. R. Masullo, M. Panniello, V. G. Vaccaro, A. Andreone, E. Di Gennaro, F. Francomacaro, G. Lamura, V. Palmieri, and D. Tonini, in Proceedings of the 10th European Particle Accelerator Conference, Edinburgh, Scotland, 2006, paper MOPCH167 (EPS-AG, Edinburgh, Scotland, 2006), p. 454.

[27] E. I. Simakov, W. B. Haynes, M. A. Madrid, F. P. Romero, T. Tajima, W. M. Tuzel, C. H. Boulware, and T. L. Grimm, First High Power Test Results for $2.1 \mathrm{GHz}$ Superconducting Photonic Band Gap Accelerator Cavities, Phys. Rev. Lett. 109, 164801 (2012).

[28] E. I. Simakov, S. A. Arsenyev, W. B. Haynes, D. Yu. Shchegolkov, N. A. Suvorova, T. Tajima, C. H. Boulware, and T. L. Grimm, Raising gradient limitations in $2.1 \mathrm{GHz}$ superconducting photonic band gap accelerator cavities, Appl. Phys. Lett. 104, 242603 (2014).

[29] J. D. Joannopoulos, S. G. Johnson, J. N. Winn, and R. D. Meade, Photonic Crystals. Molding the Flow of Light, 2nd ed. (Princeton University, Princeton, NJ, 2008).

[30] H. Padamsee, J. Knobloch, and T. Hays, RF Superconductivity for Accelerators, 2nd ed. (Wiley-VCH, New York, 2008).

[31] E. I. Simakov, S. S. Kurennoy, J. F. O'Hara, E. R. Olivas, and D. Yu. Shchegolkov, Optimizing the configuration of a superconducting photonic band gap accelerator cavity to increase the maximum achievable gradients, Phys. Rev. ST Accel. Beams 17, 022001 (2014).

[32] S. A. Arsenyev and E. I. Simakov, The design of a five-cell superconducting RF module with a PBG coupler cell, AIP Conf. Proc. 1507, 425 (2012).

[33] Computer Simulation Technology, CST Particle Studio, http://www.cst.com.

[34] ANSYS, Ansys Inc., http://www.ansys.com.

[35] T.P. Wangler, $R F$ Linear Accelerators, 2nd ed. (Wiley-VCH, New York, 2008).

[36] S. G. Johnson, http://math.mit.edu/ stevenj/18.369/pml.pdf.

[37] TESLA Technical Design report, edited by R. Brinkmann et al., DESY, 2001.

[38] A. L. Reimann and C. Kerr Grant, III. Some hightemperature properties of niobium, Philos. Mag. 22, 34 (1936).

[39] S. A. Arsenyev, W. B. Haynes, D. Yu. Shchegolkov, E. I. Simakov, T. Tajima, C. H. Boulware, T. L. Grimm, and A. R. Rogacki, High gradient testing of the five-cell superconducting rf module with a PBG coupler cell, in Proceedings of SRF2015, Whistler, Canada, paper WEA2A02 (TRIUMF, Vancouver, 2015), http:// srf2015proc.triumf.ca/prepress/papers/wea2a02.pdf.

[40] S. A. Arsenyev, R. J. Temkin, W. B. Haynes, D. Yu. Shchegolkov, E. I. Simakov, T. Tajima, C. H. Boulware, T. L. Grimm, and A.R. Rogacki, Cryogenic testing of the $2.1 \mathrm{GHz}$ five-cell superconducting RF cavity with a photonic band gap, Appl. Phys. Lett. 108, 222603 (2016).

[41] D. A. Goldberg and R. A. Rimmer, in Proceedings of the 15th Particle Accelerator Conference, PAC-1993, 
Washington, DC, 1993 (IEEE, New York, 1993), Vol. 2, p. 871.

[42] E. L. Ginzton, Microwave Measurements (Literary Licensing, LLC, Whitefish, 2012).

[43] S. Belomestnykh, I. Ben-Zvi, Y. Hao, V. Litvinenko, V. Ptitsyn, and $\mathrm{W}$. $\mathrm{Xu}$, On the frequency choice for the
eRHIC SRF LINAC, in Proceedings of IPAC 2014, Dresden, Germany, paper TUPME084, (CERN, Geveva, 2014), p. 1547, http://accelconf.web.cern.ch/AccelConf/ IPAC2014/papers/tupme084.pdf.

[44] G. V. Stupakov, Report No. SLAC-PUB-8683, 2000. 